\title{
Finite Element Modeling Approach for Optimal Electrode Configuration in Atrial Pacing
}

\author{
V Krasteva \\ Centre of Biomedical Engineering - Bulgarian Academy of Science, Sofia, Bulgaria
}

\begin{abstract}
A detailed two-dimensional finite element model of the conductive anatomy of the human thorax was designed to assess the current density distribution in atrial pacing. A genetic algorithm was developed to examine the optimal electrode configuration for tolerable esophageal pacing in three cases.

According to the model, the most appropriate bipolar electrode position is to encompass the atrium-esophagus contact length, at the level of the T7 and T8 vertebrae. The interelectrode spacing was not critical - 18 to 29 $\mathrm{mm}$. The active electrode length was adequate in the range of 11 to $15 \mathrm{~mm}$. In unipolar eso-thoracic configuration, the position of the external chest electrode did not affect the conditions for tolerable pacing. In external anterior-posterior pacing, the front electrode should be at a level above the apex and the back one - at the level of T5-T6 vertebrae. The insertion of a metal passive electrode in the esophagus resulted in more than twofold increase of the maximum current density in the atrium.
\end{abstract}

\section{Introduction}

The relatively wide acceptance of noninvasive transesophageal and transchest atrial pacing in the diagnosis and therapy of atrial arrhythmias is mainly due to the simple technique and reduced patient discomfort. Many clinical investigations were focused on lowering the stimulation threshold by examining appropriate stimulation pulse parameters and electrode positioning [1-5]. Usually the optimal electrode position for atrial pacing is taken at the lead insertion depth where maximum P-wave amplitude of the esophageal ECG was obtained. However, it could be said more about the influence of the electrode size, interelectrode distance and thorax geometry on the pain sensations in temporary stimulation.

The present work was aimed at computer simulation of the current density distribution profile in the thorax saggital plane for various electrode configurations. A genetic algorithm was implemented to examine the optimal electrode configuration for tolerable atrial pacing, using adequate optimization criteria.

\section{Method}

\subsection{Finite element model}

In this study a central saggital X-ray CT scan of a human thorax (figure 1a) was used to design a detailed two-dimensional finite element model (2D FEM) of the thoracic conductive anatomy (figure 1b). Biological tissues were considered linear, isotropic, continuous, electroconductive media with no magnetic properties. The corresponding electrical resistivities, compiled from several sources $[6,7]$ are summarized in table 1 .

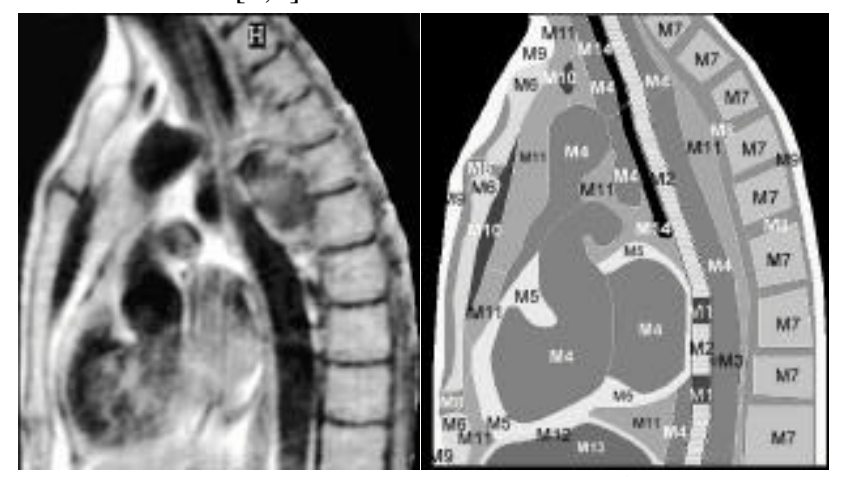

(a)

(b)

Figure 1. (a) - Central saggital X-Ray scan of the human thorax (b) - 2D FEM model with fourteen tissue conductivities.

Table 1. Specific resistivities assigned to FEM regions.

\begin{tabular}{lll}
\hline Region & Resistivity $[\Omega . \mathrm{m}]$ & Mark \\
\hline Metal electrode & $2.10^{-7}$ & M1 \\
Isolation & $2.10^{7}$ & M2 \\
Esophagus & 4 & M3 \\
Blood & 1.5 & M4 \\
Myocardium & 3 & M5 \\
Chest wall & 20 & M6 \\
Back bone & 160 & M7 \\
Intercostal muscles & 7 & M8 \\
Skin & 20 & M9 \\
Lungs & 12 & M10 \\
Fat & 22 & M11 \\
Diaphragm & 4 & M12 \\
Stomach & 7 & M13 \\
Internal air & 500 & M14 \\
\hline
\end{tabular}


The finite element mesh generated for this model consists of 21,100 nodes, defining 20,150 linear fournoded plane elements. In order to build a relatively simple and flexible system for esophageal electrode configuration studies, the esophagus region was divided into $1 \mathrm{~mm}$ height cross-section sub-areas, with possibility to change the element material properties and electric boundary loads on every solution step. By analogy, the front and back thoracic surfaces were also divided in 5 $\mathrm{mm}$ linear sectors for defining the external electrode positions.

A voltage source applied between the electrodes was used to simulate stimulus. The FE method was applied to solve the pseudo-elliptic differential equation of Laplace type with respect to the scalar electrical potential $(V)$ :

$$
\nabla(\gamma \nabla V)=0
$$

in a non-homogeneous medium with conductivity tensor $\gamma$. Equation (1) was solved under the Dirihlet and Neumann boundary conditions, applied for the electrodes and thorax surface. The current density distribution was defined by the potential gradient, using the relation:

$$
J(x, y)=\gamma(-\operatorname{grad} V(x, y)) .
$$

The real pacing pulse generates a non-stationary electrical field, but the steady state solution obtained here did not imply significant errors due to the weakly conductive body structures and low frequencies, resulting in negligible eddy current feedback reaction.

Three electrode configurations were studied, operating with the following design variables:

- electrode length, interelectrode distance and catheter insertion depth of a bipolar esophageal electrode;

- size and insertion depth of a unipolar esophageal electrode, and an external electrode position in esophageal-thoracic configuration;

- thoracic anterior-posterior electrode placement, combined with size and position of an additional passive esophageal electrode.

Since the problem is multi-parameter dependent, it requires solution involving optimization methods. The optimization criterion considered for tolerable atrial pacing is defined as:

$$
J_{\text {atrium }}^{\max } / J_{\text {esophagus }}^{\max } \Rightarrow \max \text {. }
$$

It evaluates the maximum current density in a small (5\%) fraction of the atrium area (for adequate stimulation) and the maximum current density averaged over $1 \%$ of the esophagus elements (minimum possible injury of the esophagus).

\subsection{Genetic algorithm}

A Genetic Algorithm (GA) [8] seemed convenient for solving the multi-objective optimization problem. Additional advantage of GAs is that they are not "blackbox" methods and can be controlled and monitored during the process of generation of solutions.
Genetic algorithms present the solutions of a given problem as competing individuals during genetic evolution. The solutions are obtained for different values of design variables considered as genes of those individuals. In each cycle of the algorithm (see figure 2) a subsequent generation is created by means of genetic operations. In the classical definition of GA there are two types of operations: mutations and crossovers. The idea of mutation operations is to select a particular bit of information and to alter it. By analogy with nature, the crossover operations combine the genes of two individual's chromosomes. In the process of evolution, "better" chromosomes generate larger offspring number, thus having higher chances of survival.

Our task involved a classical GA, where the design variables shown in section 2.1, specific for each electrode configuration, were encoded as genes. The individuals created by these genes are used as input parameters for the FE model for estimation of the object function (3). The evolution process was stopped after a fixed number of steps or when repeatedly similar individuals remained predominant in the overall population. To avoid the error of finding a local extremum of the object function, the algorithm was started every time with a random initial population of genes.

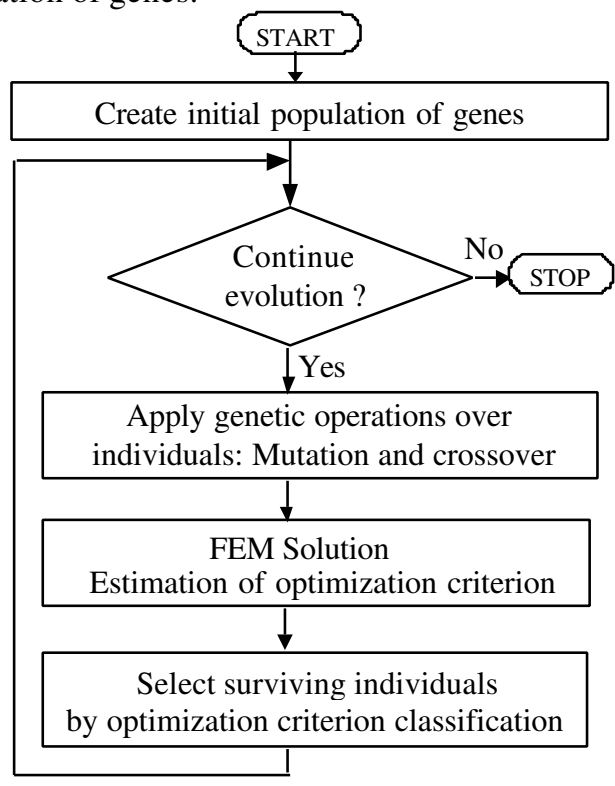

Figure 2. Genetic algorithm flowchart

\section{Results}

Since the optimization criterion defined evaluates a ratio between two quantities of equal dimension, the task was solved using the relative values of the voltage loads and resultant current densities, regardless of the real stimulation thresholds.

In bipolar esophageal configuration the maximum ratio between the atrial and esophageal current density 


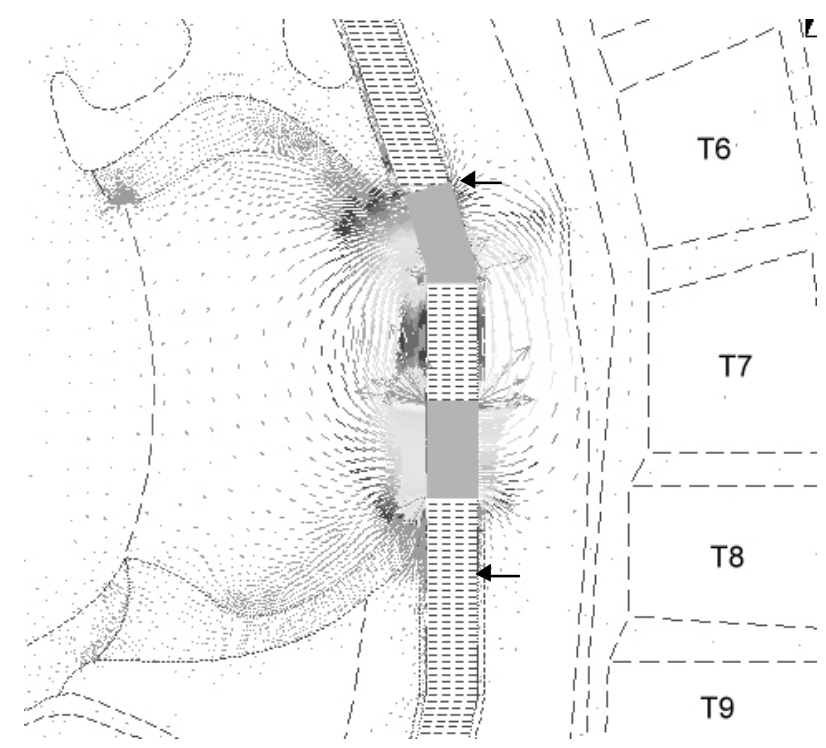

(a) Bipolar esophageal electrode configuration

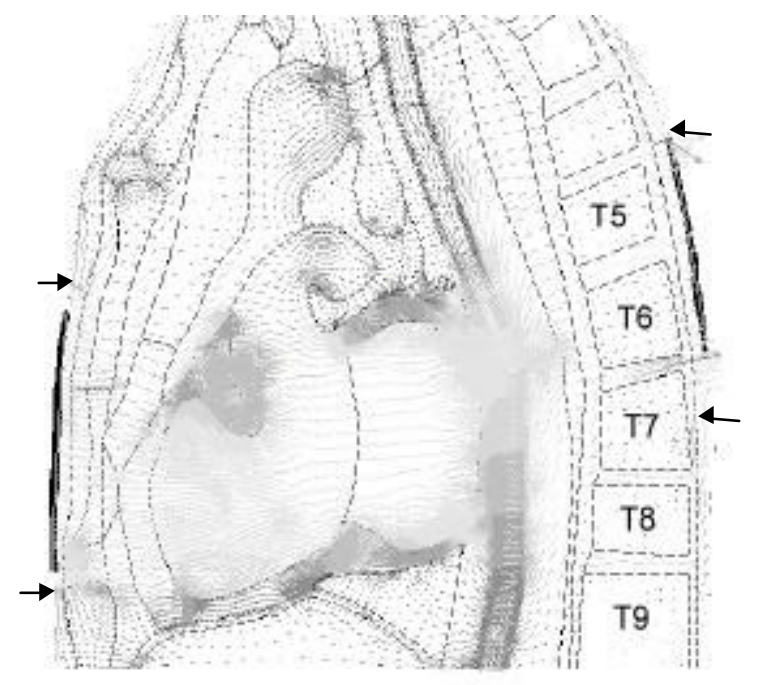

(c) - thoracic anterior-posterior electrode configuration

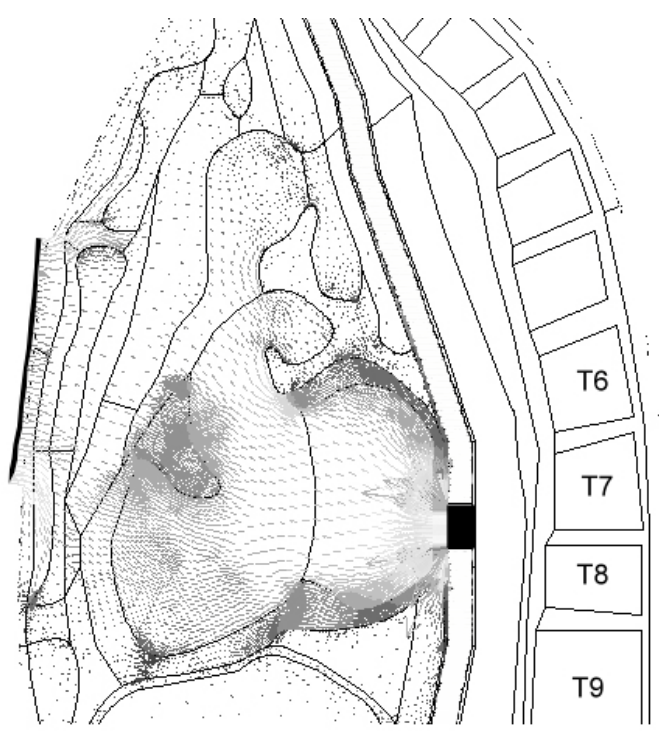

(b) Esophageal-thoracic configuration

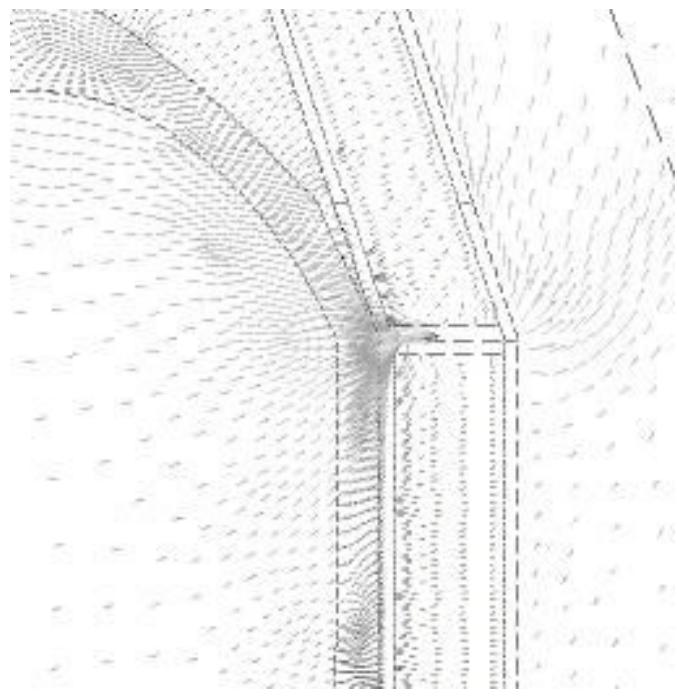

(d) - the same as case (c) zoomed at the region of insertion of an additional passive esophageal ring electrode
e respective optimal electrode configurations

In thoracic anterior-posterior electrode configuration the optimization criterion was changed. The maximum current density averaged over $5 \%$ of the atrium elements was taken. The results showed that the front electrode should be at a level above the apex and the back electrode - at the level of T5-T6 vertebrae (figure 3c). Possible electrode positions are enclosed between the arrows. For an arbitrary chosen inter-electrode potential difference of $100 \mathrm{~V}$, the maximum current density in the atrium was $J_{\text {atrium }}^{\max } \approx 81 \mathrm{~A} / \mathrm{m}^{2}$. The insertion of a passive metal electrode in the esophagus (figure $3 \mathrm{~d}$ ) increased about 3.6 times the maximum current density in the atrium 
$\left(J_{\text {atrium }}^{\max } \approx 288 \mathrm{~A} / \mathrm{m}^{2}\right.$ ). However, the esophageal metal electrode resulted also in? corresponding increase of the esophagus current density. It was $160 \mathrm{~A} / \mathrm{m}^{2}$ without the additional electrode and $630 \mathrm{~A} / \mathrm{m}^{2}$ with a $2 \mathrm{~mm}$ length electrode (figure $3 \mathrm{~d}$ ).

\section{Discussion and conclusion}

Transesophageal pacing systems take advantage of the anatomical contact between esophagus and left atrium for obtaining minimum pacing threshold. It was shown that the catheter insertion depth was closely correlated with the atrium-esophagus contact area. The inter-electrode distances found to be in the range of 18 to $29 \mathrm{~mm}$, resulted in bipolar electrode configuration encompassing the area of the atrium-esophagus contact. These values are in agreement with the clinical results of Gallagher et al. [1], Benson et al. [2] and Pehrson et al. [4]. The active esophageal electrode length should be not less than 10 $\mathrm{mm}$. A shorter electrode was associated with higher current density peaks at the electrode edges. This may result in pain sensation and possible esophageal burns. The maximum electrode diameter and length were taken in relation to anatomical considerations and catheter flexibility.

The chest electrode vertical position was found not critical for thoracic-esophageal atrial pacing. The correct unipolar esophageal electrode placement was the main determinant for high current density induction in the atrial region.

In anterior-posterior transcutaneous electrode configuration the front electrode should be at a level above the apex and the back electrode - at the level of T5-T6 vertebrae. This approach for pacing resulted in more uniform current distribution in the atrial region. However, the current density was higher in the chest wall tissues - the well-known effect of skin receptor excitation and intercostal muscles contraction, before reaching the pacing threshold. The insertion of an additional passive metal electrode in the esophagus was aimed at increasing and focusing the current density in the atrial region. However, the usual negative point was a respective increase of the current at the electrode edges. A compromise could be reached between the electrode length and the increase of the atrial and esophageal current density. It seemed reasonable to choose a longer electrode, for example 5-6 mm, instead for example $2 \mathrm{~mm}$. The shorter electrode is associated with 3.6 times higher current density in the atrium and 3.9 times higher esophageal current density. The longer electrode resulted in 3.2 and 2.9 times increase of the current density in the atrial and esophageal region, respectively.

The genetic algorithm appeared convenient and simple approach for searching solutions in electrode optimization with FEM. In our case, the genetic evolution of electrode configurations encoded by three or four design variables, usually requires no more than 150 steps. Regardless of the random choice of the initial population of genes in every new optimization cycle, the solution is convergent and repeatable.

A limitation of this study is the use of a 2D model, while $3 \mathrm{D}$ would be potentially more accurate. However, the possibility of iterative variation of electrode parameters with 3D model seemed very difficult and time consuming. Although a specific case was considered here, based on the thorax geometry of an adult patient, the accuracy of the method for electrode configuration optimization could be considered adequate. The model was applicable for approximate and quick assessment of the current distribution in the atrial, esophageal and thoracic regions and for the design of optimized electrode configurations. Possible future model development could include time-dependent analysis of the effect of stimulus waveform and duration.

\section{References}

[1] Gallagher JJ, Smith WM, Kerr CR. Esophageal pacing: A diagnostic and therapeuthic tool. Circulation 1982; 65:336.

[2] Benson D, Sanford M, Dunnigan A, Benditt DG. Transesophageal atrial pacing threshold: role of interelectrode spacing, pulse width and catheter insertion depth. Am J Cardiol. 1984;53:63-67.

[3] Res JC, Van Woersem RJ, Dekker E, Dunning AJ. Transesophageal atrial pacing - stimulation and discomfort thresholds: the role of electrode configuration and pulse width. PACE. 1991;14:1359-1366.

[4] Pehrson S, Wedekind T, Madsen B, Holm M, Res J, Olsson B. The optimal oesophageal pacing technique - the importance of body position, interelectrode spacing, electrode surface area, pacing waveform and intraoesophageal local anaesthesia. Scand Cardiovasc J 1999; 33:103-9.

[5] McEneaney D. An esothoracic electrode for electrophysiological studies. J Electrocardiol. 2002; 35:151-157.

[6] Panescu D, Webster JG, Tompkins WJ, Stratbucker RA. Optimization of transcutaneous cardiac pacing by threedimensional finite element modelling of the human thorax. Med.\&Biol.Eng.\&Comp. 1995;33:769-775.

[7] Camacho M, Lehr JL, Eisenberg SR. A three-dimensional finite element model of human transthoracic defibrillation: paddle placement and size. Trans. Biomed. Eng. 1995;42:572-78.

[8] Man K, Tang K, Kwong S. Genetic Algorithms: Concepts and Designs. London: Springer - Verlag 1999.

Address for correspondence:

Vessela Krasteva, PhD

Centre of Biomedical Engineering

Bulgarian Academy of Science

acad. G. Bonchev str. bl.105, Sofia 1113, Bulgaria

e-mail vessika@clbme.bas.bg 